\title{
Erratum: Velocity of a Perturbation in Infinite Lattice Systems ${ }^{1}$
}

\author{
C. Marchioro, ${ }^{2}$ A. Pellegrinotti, ${ }^{2}$ M. Pulvirenti, ${ }^{3}$ and L. Triolo ${ }^{4}$
}

Received February 14, 1980

The proof to Theorem 5.3, page 509, is incorrect. This theorem is only a remark to the main results of the paper, which remain correct. We apologize to the reader for this error.

1 This paper appeared in J. Stat. Phys. 19 (5), 499 (1978).

${ }^{2}$ Instituto Matematico dell'Università di Camerino, Camerino (MC), Italy.

${ }^{3}$ Istituto Matematico dell'Università di Roma, Rome, Italy, and Istituto Matematico dell'Università dell'Aquila, L'Aquila, Italy.

${ }^{4}$ Istituto Matematico dell'Università di Camerino, Camerino (MC), Italy, and Istituto di Matematica Applicata, Facoltà di Ingegneria dell'Università di Roma, Rome, Italy. 